\title{
Die Cannabis-Diskussion aus der Perspektive von Prävention und Suchthilfe
}

\author{
The Cannabis Discussion from the Perspective of Prevention and \\ Addiction Support
}

\section{Zusammenfassung}

Nach jahrelanger Stagnation der Cannabis-Politik entstand in Deutschland mit den diesbezüglichen Verfassungsgerichtsurteilen von 1994, 2002 und 2004 Bewegung. Zwar ähneln sich die Fronten der Befürworter einer wie auch immer gearteten sog. Legalisierung einerseits sowie der unveränderten oder verschärften Repression andererseits nach wie vor (obgleich die SPD-Fraktion im Deutschen Bundestag von jener zu dieser gewechselt hat). Doch wenn schon deren Diskussion nicht, so hat die Judikative Fakten geschaffen, die von gesellschaftlicher Normentwicklung beeinflusst auch gesellschaftliche Normen beeinflussen. Unter dem Strich verlangt das Verfassungsorgan einen nüchternen Umgang mit dem Rauschmittel. Solche Sachlichkeit könnte die Möglichkeiten von Prävention, Frühintervention und Therapie befördern, was angesichts steigender Konsumenten-/-innenzahlen mehr als dringlich erscheint.

\section{Schlïisselwörter}

Cannabis · Therapie · Prävention · Rechtslage

\section{Abstract}

After years of stagnation, cannabis politics in Germany were set in motion by the decisions of the constitutional court in 1994 2002 and 2004. It is true that there has not been much movement neither in the ranks of the supporters of any kind of legalisation nor among the supporters of consistent and tight repression (although the SPD faction in the German Parliament changed sides). Though these discussions did not come to anything, the judiciary created facts that were influenced by the development of norms in society, which, in their turn, will influence the norms of society. In essence, the constitutional court requires a rational handling of the drug. Objectivity might be able to promote prevention, early detection and treatment, which is an urgent requirement in view of the increasing numbers of cannabis users.

Key words

Cannabis · therapy $\cdot$ prevention $\cdot$ legal situation
Von den Implikationen der Cannabis-Urteile des Bundesverfassungsgerichts blieben indes Öffentlichkeit, Politik und auch manche Experten bislang weitgehend unberührt. Cannabis, so ist bisweilen zu hören, sei heute eine völlig andere Substanz als noch vor Jahren, seine Wirkung und Verbreitung erschreckender als jene von Alkohol und Tabak, eine ganze Generation sei in Ge- fahr. So schwang sich gar das längst verblasste Hamburger Nachrichtenmagazin vor Jahresfrist mit dem Titel „Die Seuche Cannabis“ in nicht eben luftige intellektuelle Höhen.

Von der anderen Seite hingegen haben wir etwa ernstlich zu hören, den jährlich ca. 200000 durch Tabak- und Alkoholkonsum 
vorzeitig Verstorbenen stünde kein einziger „Cannabis-Toter“ entgegen - ein Irrtum, der nur auf vollständiger Vernachlässigung der Gefahren berauschter Teilnahme am Straßenverkehr gründen kann.

\section{Epidemiologie}

Betrachten wir empirische Fakten, so müssen wir feststellen: Der Cannabiskonsum ist national wie international unter jungen Menschen über die vergangenen Jahre und Jahrzehnte gestiegen. In Deutschland betrifft dies insbesondere die Gruppe der älteren Teenager. Dabei bestehen kaum noch Unterschiede zwischen Ost und West, wohl aber zwischen den Geschlechtern. Ein geringerer Anteil der Mädchen konsumiert Cannabis und dieser dann auch durchschnittlich weniger als Jungen.

Konkret stellt Cannabis die drittmeist konsumierte psychoaktive Substanz in Deutschland dar, und unter den illegalen die mit Abstand beliebteste. Unlängst wurde erhoben, dass $31 \%$ der 12 - bis 25-Jährigen Cannabis-Erfahrung besitzen (Alkohol: 90\%, Tabak: $66 \%$ [1], d. h. zumindest einmal konsumiert haben. Auch beim aussagekräftigeren Datum des aktuellen Konsums sind die Verhältnisse identisch: Im vergangenen Jahr konsumierten $3 \%$ der 12- bis 25-Jährigen insgesamt häufiger als 10-mal Cannabis, $69 \%$ konsumierten monatlich oder häufiger Alkohol und es wurden $35 \%$ ständige bzw. gelegentliche Raucher gezählt [1]. Ein entsprechendes Bild liefern zudem die Daten des durchschnittlichen Alters beim Erstkonsum: Cannabis: 16,4 Jahre, Alkohol: 14,1 Jahre, Tabak: 13,6 Jahre.

Wiewohl also Cannabis die meistkonsumierte illegale Substanz darstellt (97,2\% aller Konsumenten/-innen illegaler Substanzen konsumieren Cannabis) [2], sprechen die Therapiezahlen eine entgegengesetzte Sprache. Zwar stiegen die registrierten Cannabis-Therapien (Hauptdiagnosen) in Deutschland über die vergangenen Jahre auf mittlerweile 13422 , doch ist ihr Anteil an der Gesamtzahl aller Therapien zum Missbrauch illegaler Drogen mit ca. 30\% (inkl. Alkohol: ca. 10\%) sehr unterproportional. (Bei ca. $40 \%$ aller Cannabis-Klienten/-innen wird „schädlicher Gebrauch“, bei ca. $60 \%$ „Abhängigkeit“ diagnostiziert.)

Erwartungsgemäß werden diese Daten von den eingangs genannten Akteuren völlig unterschiedlich gewichtet: Betonen die einen die steigenden Absolutzahlen von Cannabis-Konsum und -Therapie, so weisen die anderen auf deren unterproportionale Anteile im therapeutischen Bereich hin. Unerfreulich ist es in diesem Zusammenhang festzustellen, dass nach mehr als vierzig Jahren der neuerlichen Verbreitung von Cannabis in Deutschland selbst die absurden Extrempositionen immer noch lautstark vertreten werden. Vor diesem Hintergrund kann selbst folgende sachlich selbstverständliche - Aussage immer noch Anlass zur hitzigen Diskussion geben: Cannabis wird von der großen Mehrzahl aller Konsumenten/-innen maßvoll gehandhabt. Für diese Gruppe vorwiegend junger Erwachsener scheint Cannabis keine Substanz zu sein, deren - auch Jahre währender - Konsum Schäden hinterlassen würde. Für die weit überwiegende Mehrzahl (> 95\%) aller Konsumenten/-innen ist der Cannabiskonsum ein vorübergehendes, an Jugend und junges Erwachsenenalter ge- bundenes Phänomen. Mit fortschreitenden Lebensjahren ( $a b$ spätestens 30) wird der Konsum regelmäßig und ohne therapeutische Interventionen eingestellt.

Des ungeachtet geht Cannabis für eine prozentual zwar geringe, absolut jedoch große Zahl von Konsumenten/-innen mit gravierenden bis sehr schweren Problemen einher. Dies gilt auch jenseits des als Konsumfolge nicht belegten amotivationalen Syndroms oder der immer wieder reanimierten „Flashback“-Schimäre. Längst wissen wir - auch für Cannabis - sicher: Je früher der Konsum psychoaktiver Substanzen beginnt, desto größer ist das Risiko starken und dauerhaften Missbrauchs und einer Abhängigkeit sowie das Risiko schwerer Beeinträchtigungen und Schädigungen. Die Wahrscheinlichkeit des erfolgreichen Ausstiegs sinkt im Gegenzug. Die Europäische Beobachtungsstelle für Drogen und Drogensucht stellt hierzu fest, bei den Klienten/-innen in Cannabis-Therapie handele es „sich vorwiegend um junge Männer, die als Jugendliche mit dem Konsum von Cannabis beginnen (häufig vor dem 15. Lebensjahr)“ [3].

\section{Maßnahmen}

Angesichts dieser gesundheitspolitischen Herausforderung ersten Ranges ist nach aktuellen Handlungskonzepten zu fragen [4]. Wie reagieren Politik und (Fach-) Öffentlichkeit? Welche Perspektiven werden aufgezeigt? Die Antwort ist mehr als widersprüchlich: Beklagen viele der gegenwärtig zahlreichen Verlautbarungen zum Thema wortreich die zunehmende Verbreitung des Cannabis-Konsums unter jungen Menschen, so wird doch als Reaktion auf die so dargestellte „enorme Gefahr/Bedrohung/Seuche" oft reflexartig insbesondere die Aufrechterhaltung eben jener Maßnahme gefordert, die sich nicht gerade als wirksam erwiesen hat. Mit einem Satz: Der Konsum steigt - das Verbot muss bleiben. Weitere Konzepte - meist Fehlanzeige. Die Herausforderung Cannabis hingegen, und das macht die Entwicklung über viele Jahre national wie international deutlich, braucht differenziertere Antworten und kontinuierliches Engagement in zumindest dreifacher Hinsicht:

\section{Prävention}

Cannabis-Konsum wurde seit den 60er-Jahren des vergangenen Jahrhunderts von offizieller Seite nicht übertrieben sachlich gewürdigt (vgl. Abb. 1). Die Behauptungen seitens des Mainstreams aus Politik und Polizei deckten sich dabei weder mit den überwiegenden individuellen noch mit den Generationserfahrungen. Mit dieser Diskrepanz einher geht selbstredend ein inzwischen schon traditioneller Glaubwürdigkeitsverlust vorgeblicher Cannabis-,Prävention“ unter der Zielgruppe junger Konsumenten/ -innen. In diesem Zusammenhang war es vermutlich wenig hilfreich, zunächst mehreren Generationen junger Menschen zu vermitteln, Cannabis sei eine per se sehr gefährliche Droge, um nach Jahrzehnten dann mit der Behauptung umzuschwenken, „früher“ sei sie zwar einigermaßen harmlos gewesen, doch nun ginge von ihr tatsächlich größte Gefahr aus. (Dabei soll hier die immer wieder aufgeworfene Frage unbedingt undiskutiert bleiben, ob die Androhung von Gefahr - oder Strafe - gerade auf junge Menschen nun größere Präventiv- oder aber Anziehungskraft ausübt.) 

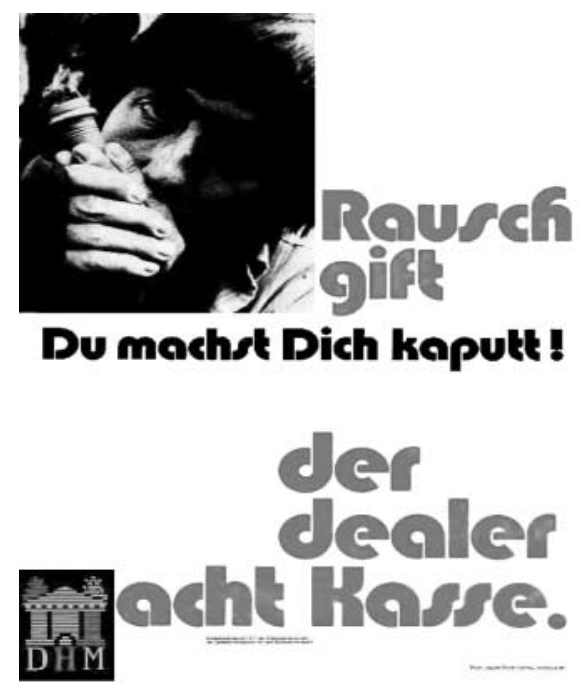

Abb. 1 „Rauschgift - Du machst Dich kaputt! der dealer macht Kasse.“ „Präventions“kampagne der Kriminalpolizei, 1971.

Die Situation ist also gekennzeichnet von einem nach wie vor eklatanten Widerspruch zwischen den Ebenen des Normativen und des Faktischen, der sich längst zum Dilemma entwickelte: Da rund ein Drittel, in Großstädten ein noch deutlich höherer Anteil junger Menschen, Cannabis-Erfahrung besitzt, senkt die pauschale Verurteilung jeglichen Konsums die Glaubwürdigkeit von Prävention gegen Null. Subjektiv machen prozentual nur wenige junge Menschen nachhaltig negative Erfahrungen mit der Substanz. Das faktische Konsumverbot wird von den meisten Konsumenten/-innen vor diesem Hintergrund nicht nachvollzogen. Immer wieder und nachdrücklich verweisen junge Menschen auf die gesellschaftlich und juristisch sehr unterschiedliche Behandlung von Alkohol und Cannabis, die auch durch das Gefährdungspotenzial beider Substanzen nicht gerechtfertigt sei. Sie erleben, dass die älteren Generationen „ihre“ Droge Alkohol protegieren und die „Jugenddroge“ Cannabis sanktionieren.

Dies legt nahe, als Basis mehr erfolgreicher statt vorgeblicher Cannabis-Prävention dessen Konsum nicht ausnahmslos, grundsätzlich und ohne Einschränkung als hochriskant zu negieren, sondern als verbreitete Erfahrung vorauszusetzen. Ein Vorgehen, das hinsichtlich Alkohols selbstverständlich ist, bei Cannabis jedoch in beschleunigten Konflikt mit den $§ \S 29-32$ des Betäubungsmittelgesetzes führt.

Vor diesem problematischen Hintergrund muss eine engagierte Prävention, auch auf das Rauschmittel Cannabis bezogen, kontinuierlich, flächendeckend und multimedial die Zielgruppe der Jugendlichen erreichen, statt sie zu befremden. Als Beispiel flächendeckender Kampagnen mag die HIV-Prävention der Bundeszentrale für gesundheitliche Aufklärung (BZgA) gelten; einen Weg der Cannabis-(Sekundär-)Prävention in Übereinstimmung mit Konsumrealitäten demonstriert Jellinek aus den Niederlanden (s. Abb. 2).

\section{Therapie}

Wie gesagt: Der Konsum von Cannabis birgt gerade für Jugendliche mit hoher Konsumfrequenz erhebliche Risiken insbesondere hinsichtlich ihrer psychischen Gesundheit. Doch wo beginnt
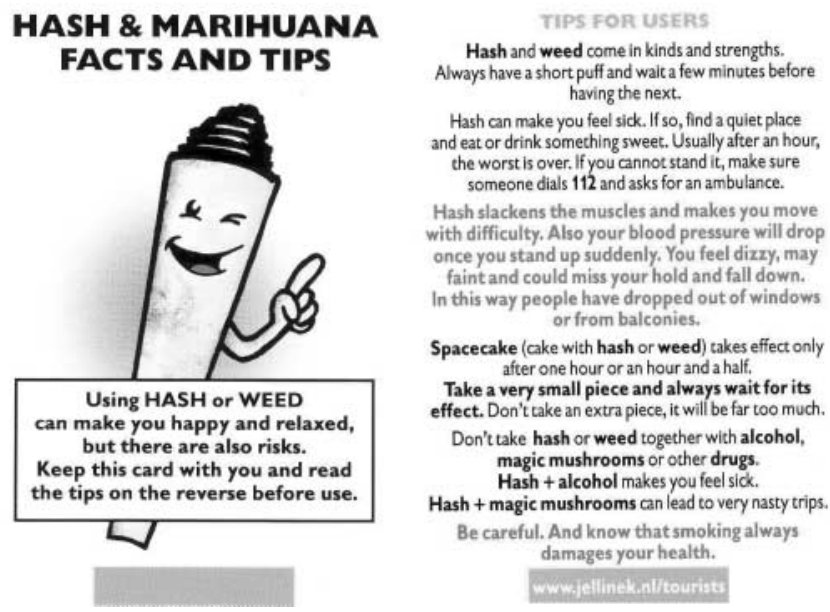

Abb. 2 Jellinek-Präventionskampagne 2004 für ausländische Touristen in den Niederlanden. Kleiner Merkzettel zur Mitnahme an der Hotelrezeption.

das Risiko? Empirisch ist diese Frage beantwortet: Zur „Mittelrisikogruppe“ zählen gemäß aktueller Therapiestudien all jene, die an durchschnittlich 14,1 Tagen/Monat jeweils durchschnittlich 7-mal konsumieren. Die „Hochrisikogruppe“ stellen diejenigen dar, die an durchschnittlich 28,7 Tagen/Monat (also beinahe täglich) jeweils durchschnittlich 10,8-mal konsumieren (also ca. 11 Joints etc.) [5].

Solch exzessivem Konsumverhalten muss therapeutisch qualifiziert begegnet werden. Allein schon vor dem Hintergrund sehr divergierender Konsumgewohnheiten zwischen Jungen und Mädchen (80\% der Cannabis-Klienten sind männlich [6], sie konsumieren tendenziell häufiger und bei jeder Konsumgelegenheit mehr als Mädchen) sollten entsprechende Angebote z.B. geschlechtsspezifisch durchdacht sein. Grundsätzlich haben sich alle Interventionen an den Kriterien von Effizienz und Effektivität messen zu lassen, d. h. auch, dass neben den Konsumintensitäten nicht zuletzt die Interventionsintensitäten der Beachtung bedürfen.

Sowohl die Zielgruppe der etwa 12- bis 20-Jährigen als auch jene der Cannabis-Konsumenten/-innen insgesamt befand sich jahrzehntelang aus verschiedenen Gründen nicht eben im Fokus des Beratungs- und Behandlungssektors. In neuerer Zeit jedoch unternimmt die Suchthilfe in Deutschland wesentliche Schritte, sich den jungen Cannabis-Konsumenten/-innen zuzuwenden. So befanden sich im Jahr 2003 mehr als 13000 Klienten mit primärem Cannabisproblem in ihrer Behandlung. Eine Analyse dieser Maßnahmen zeigte Optimierungsmöglichkeiten v. a.:

- in der Diagnostik;

- in intensiver Kooperation mit der Jugendhilfe und

- in differenzierter Behandlung [5].

Die Suchtkrankenhilfe bedarf zur Bewältigung dieser Herausforderungen wissenschaftlicher Unterstützung. Dabei können Beratung und Therapie nicht auf nordamerikanische Erfahrungen und Studien zurückgreifen, da diese in einem kaum vergleichbaren gesellschaftspolitischen Umfeld entstehen. Das Verhältnis der US- 
amerikanischen Gesellschaft zu illegalen Drogen ist juristisch, ethisch und praktisch weitgehend von europäischen Verhältnissen entfernt. Die Übernahme oder Adaption vor diesem Hintergrund entstandener Therapiemodelle scheint wenig Erfolg versprechend. Vielmehr sind dringend europäische Studien erforderlich, die beratenden und therapeutischen Zugang und Erfolgsmöglichkeiten gerade bezüglich junger Cannabis-Konsumenten/-innen aufzeigen. Wichtig sind dabei u.a. unterschiedliche Konzepte für unterschiedliche Konsumintensitäten und Begleiterscheinungen.

All dies bedarf dringend der finanziellen (Regel-)Förderung. Sie ist bislang gerade in den evident wirksamen Bereichen der Früherkennung und Frühintervention alles andere als gesichert, geschweige denn selbstverständlich.

\section{Repression}

Kurz vor Übernahme der Regierungsgeschäfte beantragte die SPD-Bundestagsfraktion, „den Eigenverbrauch (von Cannabis), soweit damit keine Fremdgefährdung verbunden ist, straflos zu stellen“. (Selbst der Abgeordnete Schily damals deutlich: „Es geht also darum, den Eigenverbrauch nicht mehr zu bestrafen.“) Das damalige Resümee der Sozialdemokraten zu ihrem Entwurf: „Die bisherige Drogenpolitik der Bundesregierung, die auf einer unausgewogenen Gesetzgebung zum Betäubungsmittelrecht beruht, ist gescheitert" [7].

Tatsächlich bestehen national wie international keine Hinweise auf evtl. gesundheitsfördernde Wirkungen der Repression des Konsums. So ist die Cannabis-Erfahrung Minderjähriger in den USA (36,5\% der 15-jährigen Jungen) trotz dort seit Jahrzehnten fundamentalistischer Verfolgung wesentlich weiter verbreitet als in den Niederlanden (24\%), die für ihre Cannabis-Politik gerade von der US-Regierung stets gescholten werden [6] (s. Abb. 3).

Gleiches gilt laut EU-Bericht auch für den Anteil der CannabisPatienten an der Gesamtzahl aller wegen Abhängigkeitsproblemen behandelten Personen: Das gegenüber Cannabis restriktive Deutschland weist hier ebenso ca. $7 \%$ auf wie etwa das deutlich liberalere Spanien.

So behauptet denn bislang auch kein einziger Politiker und kein einziger Suchtexperte, dass die Cannabisprohibition die Verbreitung oder die Konsequenzen des Konsums bislang positiv beeinflusst hätte. Stellvertretend für die meisten sei hier vielmehr die Bundesdrogenbeauftragte (aus dem Online-Forum der SPD-Fraktion im Jahr 2001) zitiert: „Ich denke [...] nicht, dass die Strafverfolgung das geeignete Mittel ist, um mit dem Problem umzugehen. [...] Wir vertreten also eine Entwicklung hin zu einer Entkriminalisierung des Cannabiskonsums in kleinen Mengen." Dennoch nennt das Bundeskriminalamt letztmalig auch für 2003 die - erneut gestiegene - enorme Zahl von 148973 erfassten Cannabis-Delikten, davon 74\% reine Konsumentendelikte [9].

Als Ersatz- und Ergänzungsstrafrecht kommt eine große Zahl an It. Bundesverfassungsgericht widerrechtlichen Fahrerlaubnisentzügen hinzu. Als Folgen sind definitiv: die soziale Ausgrenzung eines steigenden Anteils junger Menschen insbesondere

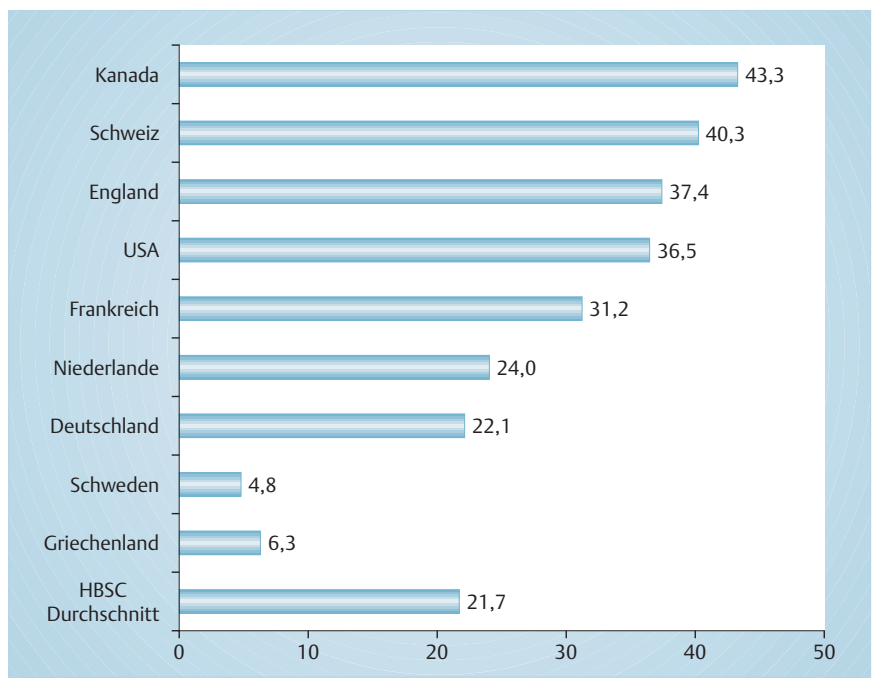

Abb. 3 Cannabis-Konsum in den vergangenen 12 Monaten unter 15-jährigen Jungen (in \%), HBSC(Health Behaviour in School-aged Children)-Studie, WHO 2004.

über den Verlust von Arbeitsplatz und Führerschein sowie selbstverständlich: Kosten für Polizei, Justiz und Strafvollzug, die sämtliche Investitionen in Prävention und Therapie bei weitem überragen. Die Konsumenten-/-innenzahl stieg hiervon unbeeindruckt.

\section{Resümee}

Zu Cannabis sind nach wie vor wenig sachdienliche Extrempositionen diskursbestimmend. Dabei wird vor allem die international ausnahmslos zunehmende Verbreitung beklagt, ohne dass man auf die Ursachenanalyse auch nur einen Gedanken verschwendet. Doch Reaktionen ohne Problemanalyse sind wenig Erfolg versprechend. So bedarf es zunächst der Erörterung dieser Frage. Wir wissen um die zentralen Gründe individuellen Cannabis-Konsums: der Wunsch nach Entspannung und Beruhigung, auch Angstbewältigung, das Vergnügen am Rausch. Es fehlen Antworten, warum diese Motive für immer mehr junge Menschen - und zwar länderunabhängig - derart reizvoll scheinen.

Sodann bedarf es einer ergebnisorientierten, nüchternen Gesundheitspolitik bezüglich des Konsums aller Rauschmittel, die auch den Konsum von Cannabis umfasst. Sie muss sich auf Erkenntnisse statt Glaubenssätze stützen. Zentraler Ansatz ist dabei, den Rauschmittelkonsum in keinerlei Hinsicht nahe zu legen oder zu erleichtern. Das bedeutet u.a. konkret:

- keine Werbung für keinerlei Rauschmittel (in vielen europäischen Ländern schon heute selbstverständlich);

- keine Rauschmittel an Automaten und Tankstellen;

- keine Subventionierung der Rauschmittelproduktion (etwa des Wein- und Tabakanbaus);

- dauerhafte, massenmediale Prävention des Rauschmittelkonsums (ähnlich der HIV-Prävention);

- erprobte, zielgruppengenaue Therapieangebote, die auch aus Alkohol- und Tabakabgaben finanziert werden. 
Grundsatz dieses Konzepts ist es, alles zu unternehmen, das nachweislich Verbreitung und Schäden effektiv mindert, sowie alles zu unterlassen, das nachweislich konsumfördernd wirkt oder wirkungsvoll ist.

Dass in diesem Zusammenhang Cannabis in einem umfassenden Sinn (inkl. Produktion und Handel) „legalisiert“ werden sollte, ist eindeutig zu verneinen. Immerhin verhieße die damit einhergehende weitere Industrialisierung wenig Gutes, wie die gesellschaftlichen Probleme mit Alkohol und Zigaretten zeigen. Die THC-Markenzigarette, womöglich noch beworben und aus Automaten erhältlich, wäre zweifelsohne ein gesundheitspolitisches Desaster ersten Ranges.

Kurzfristig realisierbar hingegen wäre die Umsetzung der Cannabis-Urteile des Bundesverfassungsgerichts - ein überfälliger Schritt, wegen des Besitzes von Cannabis zum Eigenkonsum nicht länger individuell Biografien und insgesamt jegliche Intervention zu gefährden. Hierbei böte es sich an, dem britischen Weg zu folgen: die Einstufung des Cannabis-Besitzes zum Eigenkonsum als bußgeldbewehrte Ordnungswidrigkeit. Dies wäre zugleich der definitiv geringstmögliche Identitäts- und Gesichtsverlust traditioneller Politik, es würde nicht gegen die bestehenden internationalen Verträge verstoßen - und es würde, soweit absehbar, die Konsumenten-/-innenzahlen nicht steigern.

Zu Cannabis wie zu allen übrigen Suchtstoffen ist dringend und endlich „Sachpolitik aus einem Guss“ gefragt - statt des weiteren Verharrens in Symbol-, Signal- oder Glaubenspolitik. Die Mög- lichkeiten von Prävention, Frühintervention und Therapie sind so unübersehbar wie bislang ungenutzt. Zeit, zu handeln.

\section{Literatur}

${ }^{1}$ Bundeszentrale für gesundheitliche Aufklärung. Die Drogenaffinität Jugendlicher in der Bundesrepublik Deutschland 2004. Eine Wiederholungsbefragung der BZgA. Köln: BZgA, 2004

${ }^{2}$ Kraus L, Augustin R, Orth B. Illegale Drogen, Einstiegsalter und Trends. Ergebnisse des Epidemiologischen Suchtsurveys 2003. Sucht 2005; 51 (Sonderheft 1): 19-28

${ }^{3}$ Europäische Beobachtungsstelle für Drogen und Drogensucht. Jahresbericht 2003. Stand der Drogenproblematik in der Europäischen Union und in Norwegen. Luxemburg: Amt für amtliche Veröffentlichungen der Europäischen Gemeinschaften, 2003

${ }^{4}$ Gaßmann R. Deutsche Hauptstelle für Suchtfragen. Cannabis. Neue Beiträge zu einer alten Diskussion. Freiburg i.Br: Lambertus, 2004

${ }^{5}$ Simon R. Behandlungsangebot und -nachfrage bei cannabisbezogenen Störungen. Straßburg: Deutsch-französische Tagung „Cannabis - Prävention, Behandlung, Politik“, 11.7.2005

${ }^{6}$ Perkonigg A et al. Problematischer Konsum illegaler Substanzen, Hilfesuchverhalten und Versorgungsangebote in einer Region. Suchtmedizin 2004; 6: $22-31$

${ }^{7}$ Drucksache 13/6534. Gesetzentwurf der Abgeordneten Gudrun Schaich-Walch ... Entwurf eines Gesetzes zur Änderung des Gesetzes über den Verkehr mit Betäubungsmitteln (Betäubungsmittelgesetz BtMG), 11.12.1996.

${ }^{8}$ Currie $\mathrm{C}$ et al. Young people's health in context. Health Behaviour in School-aged Children (HBSC) study. International report from the 2001/2002 survey. Copenhagen: World Health Organization, 2004

${ }^{9}$ Bundeskriminalamt. Bundeslagebild Rauschgift 2003. Wiesbaden: BKA, 2004 